\title{
MORTAR PHYSICAL PROPERTIES EVOLUTION IN EXTRUSION FLOW
}

\author{
ARNAUD PERROT \\ LGCGM équipe matériaux, INSA de Rennes \\ 20, avenue des buttes de Coesmes 35043 Rennes, France \\ arnaud.perrot@ens.insa-rennes.fr \\ CHRISTOPHE LANOS
}

LGCGM équipe matériaux, IUT de Rennes

3, rue du Clos Courtel - BP 9042235704 Rennes CEDEX 7

christophe.lanos@univ-rennes1.fr

YANNICK MELINGE

LGCGM équipe matériaux, INSA de Rennes

20, avenue des buttes de Coesmes 35043 Rennes, France

yannick.melinge@insa-rennes.fr

PATRICE ESTELLÉ

LGCGM équipe matériaux, INSA de Rennes

20, avenue des buttes de Coesmes 35043 Rennes, France

patrice.estelle@insa-rennes.fr

\begin{abstract}
Extrusion is not a common way to form firm cement-based material. Due to material frictional behaviour drainage phenomenon appears and leads to process blockage. The properties of the extrusion multiphase flow of such specific materials have to be highlighted in order to understand the properties of the process flow. An extrudible mortar is formulated according to extrusion criteria provided by literature tests are developed in order to describe the evolution of the paste undergoing extrusion. To study the relationship between flow properties, extrusion force and material consolidation, a technique based on the measurement of paste hardness in the billet zone is developed. Measurements of the local paste density and flow visualization are also performed. Results show that in the range of used ram velocities, filtration occurs between fluid and solid phases. This creates zones of preferential flow and of non-flowing consolidated material.
\end{abstract}

Key words: Ram extrusion, Firm mortar, multiphase flow

\section{Introduction}

Extrusion is a common forming process for a wide range of materials (food, polymer, clay, metal). For perfect and homogeneous plastic materials, the extrusion stress and the flow typology are well described and have been the subject of numerous studies. For cement-based materials, extrusion could be a high productivity forming process which is expected to improve the formed material mechanical properties (voids ratio reduction). However, it is not yet a common way to form cement based materials in an industrial setting. This is both 
explained by the heterogeneity (large range of grains size) and the rheological behaviour (high solid volume fraction) of such materials. This present paper is intended as a step in this direction.

Mu et al. (1999) or Peled and Shah (1999) showed that extrusion process is able to form hardened cement composites with higher mechanical properties compared with traditional casting process. Those studies focused on cement-based pastes with admixtures, such as viscosity agents or plasticizers, reinforced with different types of fibers. Such engineered cement composites present optimized mechanical properties. However their prohibitive cost limits their application in industrial settings. In order to extend the application fields of extruded cement based materials, Toutou et al. $(2004,2005)$ recently developed a method to identify the rheological behaviour of less expensive cement based materials (by limiting the use of admixtures) in order to evaluate their extrusion ability. The authors showed that extrusion ability (called extrudability) of such cement based materials strongly depends on its rheological behaviour. As example, those works showed that an extrudible material requires a sufficient yield stress to retain its shape (around $20 \mathrm{kPa}$ ). This imposes a material composition with high solid volume fractions. Squeeze tests performed by Toutou et al. $(2004,2005)$ highlight that extrudible cement based materials are very sensitive to drainage and liquid phase filtration through the granular skeleton at low shear rate. In consequence the apparent yield stress of the material is proportional to the applied pressure. Such behaviour can be modelled with a Coulomb law with a proportionality coefficient decreasing with a shear rate increase. The authors called frictional plastic such an apparent behaviour induced by the liquid phase migration. Perrot et al. (2006) showed that the drainage phenomenon and the frictional behaviour of cement pastes are primordial in order to compute the extrusion load. Such behaviour is frequently encountered with concentrated suspensions (Coussot et Ancey, 1999). The liquid filtration sensitiveness has already been observed in extrusion of high solid volume fraction ceramic and polymer pastes by Götz et al. (1993, 2002), Burbidge et al. (1995) and Li et al. (1995). The aim of this paper is to investigate the extrusion flow conditions and also evaluate the mechanisms of liquid filtration during mortars extrusion. Such investigations can not be directly performed during extrusion flow. This imposes a study of the paste remaining in the extruder after the extrusion. . Studying the local properties (composition, rheological behaviour or local hardness) of the material remaining in the extruder gives crucial information on the evolution of the extruded paste.

The influence of the ram velocity on the liquid filtration is studied. The local density of the mortar and the yield stress of the extrudates are also measured. As a result, the evolution of the paste composition is described. Finally, the influence of liquid filtration phenomenon on extrusion flow is discussed.

\section{Behaviour of firm mortars in extruder}

\section{Squeeze flow/extrusion similarity}

Based on squeeze flow/extrusion similarity, Toutou et al. $(2004,2005)$ have shown that during extrusion, concentrated cement pastes or mortars undergo a drainage process. For a given extruder, the work of Toutou et al. $(2004,2005)$ leads to the determination of the more suitable material formulation for extrusion 
flow. This work gives crucial information on the behaviour of the firm cement based paste in an extrusion-like flow.

In order to model the squeeze flow of cement pastes, these authors developed an analytical model based on a soils mechanics approach. Such analysis is based on the total stress (noted $\sigma$ ) decomposition (Terzaghi theory, as shown in eq. (1)). The total stress is assumed to be the combination of the stress acting (noted effective stress, $\sigma^{\prime}$ ) on the granular skeleton and the stress acting on the liquid phase assumed incompressible (noted excess pore pressure, $\mathrm{U}$ ).

$$
\sigma=\sigma^{\prime}+\mathrm{U}
$$

Moreover, the excess pore pressure and the effective stress depend on time. The initial pore pressure gradient induces a flow of the liquid phase through the granular skeleton. Such flow dissipates the excess pore pressure toward the reference pressure. This step represents the consolidation of the granular media. The duration of the consolidation is monitored by the hydraulic permeability of the granular skeleton. When all the excess pore pressure is dissipated, the total stress is equal to the effective stress. The fluid filtration induces local changes of the paste composition. Moreover, induced volume changes are quite imperceptible.

The analysis of Toutou assumes that the total stress gradient is close to the effective stress gradient because in the solid mass the drainage occurs quasi instantaneously. Squeeze flows performed at low velocities induce non homogeneous flows with interstitial fluid filtration through the granular skeleton. The squeezed material is drier in the central zone than in its periphery. The filtration induced a material apparent strengthening (yield stress increase). Such behaviour, modelled by the Coulomb or Drucker -Prager theory, is called frictional plastic by the authors.

According to Toutou et al. (2004, 2005),such a multiphase flow is not surprising because of the high solid volume fraction of the studied materials. This property enhances friction between grains and consequently stresses and pressure gradients which amplify the fluid filtration.

\section{Consolidation and extrusion flow relationship for concentrated cement based material}

The liquid migration and heterogeneous distribution have already been investigated in the case of ceramic extrusion flow. Li et al. (1995) have measured the moisture content along the extruder in order to quantify liquid filtration and distribution. Burbidge et al. (1995) have studied the effect of consolidation on the flow typology inside the extruder and have tried to model the extrusion stress using soils mechanic theory for consolidation. The authors observed variations in the length of the dead zone with the increase of extrusion load. Similarly to soils, the studied materials present an apparent behaviour that greatly depends on the submitted stress path. The material keeps the highest yield stress value induced by the consolidation during the material loading. 


\section{Experiments}

\section{Material and mixing protocol}

The mortar used in this study, fills all the extrudability criteria defined by Toutou et al. $(2004,2005)$. The material is sufficiently firm to retain its shape under gravity and handling. Tests were carried out on a mortar with a water/cement weight ratio of 0.25 . A water reducing admixture (Sika plastiment $22 \mathrm{~S}$ ) is added to the water in the following weight ratio: Plasticizer/Cement $=0.01$. The binder used is a mix of 70 weight percent of Portland cement 32.5 CEM IIB with 20 percent of volcanic rock (pozzolane) finely crushed $(15 \mu \mathrm{m}$ maximum particle diameter), 5 percent of silica fume $(0.1 \mu \mathrm{m}$ maximum particle diameter) and 5 percent of amorphous crushed quartz (10 $\mu \mathrm{m}$ maximum particle diameter). The addition of filler fines gives better homogeneity and plastic performance to the paste. Mortars were obtained adding fine sand (maximal diameter of grains: 0.630 $\mathrm{mm}$ ) in a weight ratio Sand/Cement $=0.8$. The final fresh mortar presents a high yield stress (about $20 \mathrm{kPa}$ ) and a high solid volume fraction $(76.8 \%$ ) paste. The protocol of mixing is given in the work of Perrot et al. (2006) and extrusion tests are performed 30 minutes after the end of mixing in order to avoid aging effects.

The yield stress of the studied material is measured with vane tests A four-bladed vane of radius $R_{i}=8 \mathrm{~mm}$ and of height $H=8 \mathrm{~mm}$ is used for the measurements. Tests performed at different rotation speed $(0.1$ to $10 \mathrm{rpm})$ give the same recorded torque-displacement curves. Consequently, we assume that the tests result and the material do not depend on shear rate: the viscous behaviour of the paste is negligible. We presently consider that when the flow is initiated the yield stress $\mathrm{K}$ is reached on the sheared surface. In consequence, a force balance yields (Steffe, 1996). The used $20 \mathrm{~cm}$ diameter cup avoids wall slip effects.

$$
\mathrm{K}=\frac{\mathrm{M}}{2 \pi \cdot \mathrm{H}_{\mathrm{R}}{ }^{2}}
$$

Where $\mathrm{M}$ is the maximal torque recorded during shearing.

For the studied extrudible mortars with a high solid volume fraction, the water content affects considerably the rheological behaviour, as shown on figure $1.1 \%$ increasing of water content w\% (water mass on solid mass ratio) leads to multiply the yield stress K by 1.7 .

\section{Figure 1}

Consequently, the study of the extrusion flow of concentrated cement pastes and mortars has to integrate the study of the paste consolidation (Perrot et al., 2006). It is a crucial point because consolidation may induce extrudate defects and an increase of the extrusion load that can lead to the process blockage.

The extruder experimental geometry is shown on figure 2 . The geometry is axisymetrical. In consequence, $\operatorname{ram}($ diameter $\mathrm{D}=43.3 \mathrm{~mm}$ ) and die (diameter $\mathrm{d}=$ $15 \mathrm{~mm}$ ) presents a circular cross section. For the present study, the die consists in an abrupt contraction (angle of the die entry equal to $90^{\circ}$ ). The mass of extruded material is equal to $600 \mathrm{~g}$. The position of the material in the billet zone is localized by the radius $r$ and the distance from the die entry 1 . 


\section{Flow Typology analysis}

The flow typology analysis is carried out using two intrusive methods based on the analysis of the material remaining in the extruder. The first one is based on local density measurements and the second one consists in measuring a consistency index. Such investigations are repeated in the same experimental conditions but for different ram displacements in order to evaluate the material evolution in the billet zone during extrusion.

The development of such techniques seems to be very interesting because the material evolution is monitored with precise observations of the multiphase flow grains/paste/liquid in the extruder.

\section{Method based on the local density evolution}

During extrusion, liquid filtration affects the local density of the material. In this case, increase of liquid content reduces the material density. For different ram displacements, the process is stopped and the exit die is removed. Then, the ram pushes the billet outside the extruder. Then, the material remaining in the extruder is extracted and cut in small $15 \mathrm{~mm}$ thickness cylindrical layers and the local density of each layer is measured by hydrostatic weight (figure 3). For two ram velocities (a "slow" one: $0.1 \mathrm{~mm} / \mathrm{s}$ and a "fast" one: $1 \mathrm{~mm} / \mathrm{s}$ ), the ram is stopped at fixed ram displacements: $0.5,1.5,3.5,5.5 \mathrm{~cm}$ and local densities are measured.

\section{Figure 3}

Such technique allows us to plot a profile along the l-axis of the mean local value of the layer density. No information is given for induced heterogeneities along the r-axis.

\section{Method based on the consistency index evolution}

A method based on the study of the evolution of a consistency index (P) is developed in order to better describe the flow-induced heterogeneity, with crucial information along the r-axis.

In order to reach such results, an experimental protocol (figure 4) is developed and allows to plot a precise 2-dimensional $(r, 1)$ map of the instantaneous material state of consolidation.

\section{Figure 4}

Firstly, the ram is stopped for a chosen ram displacement. Then, the material remaining in the extruder is removed and cut in the direction of the ram displacement in two equal parts.

The consistency index is defined as the force required for the $5 \mathrm{~mm}$ penetration of a $1.5 \mathrm{~mm}$ diameter needle. Such penetration tests are performed on a texture analyser TA-xT2 distributed by Stable Micro-System ${ }^{\circledR}$. The software controls the needle displacement at a constant velocity $(1 \mathrm{~mm} / \mathrm{s})$ and records the penetration force. The studied value is the recorded peak value.

The chosen distance between penetrations of $5 \mathrm{~mm}$ avoids fracture effects. It gives a sufficient force response and does not affect neighbouring measurements. A minimal distance of $10 \mathrm{~mm}$ between two penetrations and a minimal $5 \mathrm{~mm}$ distance from the edge of the cut sample are fixed for the same fracture reasons. Finally, a measurement grid of the local surface hardness of the paste is obtained. The needle diameter is limited to $1.5 \mathrm{~mm}$ (equal to 2.5 times the maximal 
diameter of sand grains) also to avoid sample fractures. This induced scaling effect due to the size of sand grains may appear. However, in order to avoid such measurement artefact, the obtained value is averaged on 4 measurements ( 2 on each cut parts, for the same values of $r$ and 1 ). Results show that the variation between the 4 measurements is less than $10 \%$. Moreover, by performing the same test at different extrusion time, we evaluate the material consolidation and follow the evolution of the liquid filtration. This is performed for two different ram velocities $(0.1$ and $1 \mathrm{~mm} / \mathrm{s})$ in order to check the influence of the relative water/grains velocity in the paste consolidation. For both velocities, the extrusion is stopped for fixed ram displacements: $5,15,35,50 \mathrm{~mm}$, until the process blockage.

The consistency index appears proportional to the material yield stress. To obtain such result a mortar sample is submitted to aging during 60 minutes. Vane tests and consistency index measurements were alternatively performed. The curve of yield stress K vs. consistency index is plotted (figure 5)

\section{Figure 5}

\section{Flow typology visualisation}

In order to study the effects of paste consolidation on extrusion flow typology, we extrude a billet composed of alternated bi-coloured layers. Such a product is obtained by the superposition of layers of $25 \mathrm{~mm}$ of mortars and layers of mortars with a mass substitution of carbon equal to $1 \%$ of the cement mass. We consider that the carbon do not modify the mortar behaviour. We performed two extrusion tests with a billet mass of $800 \mathrm{~g}$ and two ram velocities of 0.1 and $1 \mathrm{~mm} / \mathrm{s}$.

After a given ram advance (here $50 \mathrm{~mm}$ ), the billet is removed of the extruder and the billet is cut in the direction of the ram advance. Then, the deformations of the layers boundaries are observed and indicate the flow typology.

Combining the water filtration informations given by the hardness local density measurement and the flow typology visualization, mechanisms of material biphasic flow is explained.

\section{Yield stress measurement}

The yield stress of the extrudates is measured for every performed test. The yield stress value is representative of paste composition and liquid filtration. As previously described, it indirectly provides the energy required for the material forming because in plastic theory the instantaneous extrusion load and the instantaneous yield stress are proportional (Hill, 1948).

A yield stress measurement is performed every $5 \mathrm{~cm}$ of extrudate and an average value is computed for each test.

\section{Flow visualisation}

\section{Local density measurements}

\section{Billet}

The evolution of the local density of the material inside the extruder is studied for two different velocities $0.1 \mathrm{~mm} / \mathrm{s}$ and $1 \mathrm{~mm} / \mathrm{s}$. 
Results (figure 6a and 6b) clearly show two distinct drier areas. The first one is located around the die and constitutes the dead zone. The second one consists in a time-spreading layer located near the ram.The fluid filtration induced by the axial stress gradient between the ram and the die imposes a consolidation of the paste located near the ram. The consolidation near the die consists in the dead zone formation near the ram which is compressed by the flowing material against the extruder and die walls.

\section{Figure 6a-b}

The density profile can be modelled by two crossing lines as shown on figures $6 \mathrm{a}$ and $6 \mathrm{~b}$. Near the die, the density remains approximately constant whereas near the ram, it seems to vary linearly with the ram distance. The crossing point of those two lines represents the consolidation front. It represents the extension of the consolidated zone inside the extruder. The consolidation front moves toward the die according to the ram displacement till a critical value where it stops (For the two ram velocities the front location is the same for the last two ram displacements). The consolidating zone moves faster in the case of low ram velocities.

\section{Extrudates}

The density of the extrudates remains constant during extrusion and equals 2140 $\mathrm{kg} / \mathrm{m}^{3}$ in the case of a $1 \mathrm{~mm} / \mathrm{s}$ ram velocity and $2130 \mathrm{~kg} / \mathrm{m}^{3}$ with a $0.1 \mathrm{~mm} / \mathrm{s} \mathrm{ram}$ velocity (the paste initial density is $2180 \mathrm{~kg} / \mathrm{m}^{3}$ ). This shows that the extrudates density increases with the ram velocity.

The filtrated liquid flows through the granular skeleton and finally leaves the extruder into the extrudates. The filtration effect is more important in the case of low ram velocities because the material in the extruder is submitted to consolidation during a longer time. To conclude, the relative displacement between liquid and solid phases is higher in the case of the lowest ram velocity.

\section{Local hardness measurement}

The local density measurement provides the evolution of an average density profile along the direction of the ram displacement but do not give indications about the fluid migration in the radial direction. Especially, the localisation of the dead zone is not possible in the radial direction. The measurements of the local consistency index can provide more indications and allow drawing a precise 2 dimensional map of the material consolidation at a given ram displacement. This procedure, defined in section 3, is performed for two values of ram advance velocities: $0.1 \mathrm{~mm} / \mathrm{s}$ (Figure $7 \mathrm{a}$ to $7 \mathrm{c}$ ) and $1 \mathrm{~mm} / \mathrm{s}$ (Figure $8 \mathrm{a}$ to $8 \mathrm{~d}$ ) to evaluate the influence of the velocity on the multiphase flow and the consolidation.

\section{Figure 7a-c Figure 8a-d}

In order to examine the influence of paste consolidation on the extrusion force, the instantaneous extrusion force is given for each map of the hardness index. Two consolidated areas clearly appear: the dead zone around the die and an expending layer near the ram. 
Results show that the thickness of the consolidated zone located near the ram depends on the ram velocity. For a given ram displacement, this cylindrical consolidated layer is smaller in the case of $1 \mathrm{~mm} / \mathrm{s}$ (high velocity). It appears that the extrusion force increase is linked to the thickness of this consolidated layer. This consolidated zone location and length can be explained by the relative velocity between the granular skeleton and the liquid. For a same ram displacement, the relative displacement between fluid and grains is higher in case of low velocities because the fluid has more time to filtrate. Such flow-induced consolidation is due to the liquid phase filtration through the granular skeleton. The extruded mortar is a composite fluid that components heterogeneously flow under extrusion conditions. As shown by Darcy's law (eq. 3), the flow is monitored by the hydraulic permeability of the granular skeleton and the extrusion conditions (excess pore pressure gradient and extrusion velocity).

$\frac{4 \mathrm{Q}_{\text {fluid }}}{\pi \mathrm{D}^{2}}=\frac{\mathrm{k}}{\eta} \cdot \frac{\Delta \mathrm{P}}{\Delta \mathrm{l}}$ (3)Where $\mathrm{Q}_{\text {fluid }}$ is the quantity of fluid filtrated, $\eta$ is the

interstitial fluid viscosity, $\mathrm{k}$ is the hydraulic permeability of the skeleton and $\Delta \mathrm{P}$ is the variation of excess pore pressure. Comparison between paste consolidation and measurements of the extrudates yield stress shows that consolidation induces a migration of liquid toward the die and gives higher water content to the material located in the shearing zone (Figure 9). Finally, the formed material is located in a preferential flow zone near the die, between the two consolidated zones (as it requires less energy to be formed). A general scheme of the extrusion flow of firm cement-based material is here clearly described.

\section{Figure 10}

It is important to note that a vault of consolidated material appears in the flow (Figure 10). This phenomenon is more present in the case of a low ram velocity and can explain the reason of the extrusion blockage. It appears that when the consolidating vault meets the dead zone, it induces a large extrusion force increase. This mechanism is also reached in the case of high ram velocity but it appears later due to the slowest liquid migration velocity.

As expected, the hardness measurement technique provides an accurate localisation of dead zone. The consolidated zone near the die behaves as a forming convergent die. The mechanism of formation of the dead zone seems to be the same for the two velocities but appears at a lowest ram displacement for a ram velocity of $0.1 \mathrm{~mm} / \mathrm{s}$.

Such result permits to study the local evolution of the material rheology during extrusion in crucial billet points. According to the relationship between yield stress and consistency index, we plot the evolution of the yield stress with the ram displacements in 3 representative zones inside the extruder:

- in the dead zone (bottom left or right corner in figure 2),

- in the shearing zone (at the die entry),

- in the ram-located consolidation zone (ram center).

Such investigations illustrate the material heterogeneous evolution inside the extruder during forming process.

\section{Figure 11a to $\mathrm{C}$}

In the forming zone at the entry die, the yield stress of the material reduces till a constant value smaller than the initial one. This proves that fluid filtrates during 
the whole extrusion toward the extrudates. The fluid flow through the granular skeleton seems to remain curiously quite constant during the flow.

The observation of the yield stress evolution near the ram and in the dead zone confirms that the material consolidation is more important in case of the low velocity (for a ram displacement of $35 \mathrm{~mm}$ near the ram, the yield stress is multiplied by 4.5 at $0.1 \mathrm{~mm} / \mathrm{s}$ and by only 2.35 at $1 \mathrm{~mm} / \mathrm{s}$ ).

Moreover, the increase of the material yield stress is more important in the area near the ram. Such material consolidation in the ram area is responsible for the extrusion force increase.

\section{Impact of liquid filtration on the flow typology}

\section{Figure 12}

Here, we focus on the effect of the liquid filtration on the flow typology. As a small variation of liquid content greatly affects the mortar behaviour, we expect that the induced change of local rheology affects the flow typology. The liquid filtration (and the paste consolidation) creates heterogeneities in the billet that are expected to change the flow typology. Two tests are performed with an extrusion ratio of 0.35 and a billet mass of $800 \mathrm{~g}$ at low $(0.1 \mathrm{~mm} / \mathrm{s})$ and high $(1 \mathrm{~mm} / \mathrm{s})$ velocities (Figure 12a and 12b). Changing the ram velocity, the consolidation conditions and the relative velocity between interstitial liquid and granular skeleton and are modified and finally the typology of flow evolves.

For the high ram velocity, the sheared zone is larger than for the low ram velocity (Figure 12a and 12b). Indeed, as the consolidation is located nearer the ram in the case of high velocity, the material is more homogeneous near the die. Inversely, in the case of low ram velocity, the filtration time is longer and the consolidating zone can spread. Such a migration induces a sensitive heterogeneity of the paste inside the extruder, creating areas where the material contains more liquid and requires less energy to flow. In consequence, the plug flow concerns a larger area and the sheared flow is confined in the area very close to the exit die.

\section{Conclusions}

The mechanisms that govern extrusion flow of mortars or cement pastes is the crucial point for the development of a feasible industrial extrusion process for such materials. Specific properties of extrudible firm mortars such as heterogeneity and high solid volume fraction are obstacles for the achievement of the extrusion process. The high solid volume fractions and the extrusion low shear rate enhance conditions for liquid drainage and paste consolidation. Moreover, paste rheology is very sensitive to water content. However, in such a forming process, the paste homogeneity is a key parameter for hardened extrudates quality and process efficiency. In this study, we have investigated the biphasic flow of heterogeneous mortar in a ram extrusion configuration in order to study the paste evolution and the liquid migration inside the extruder.

Based on previous works of Toutou et al. (2004, 2005), we formulate an extrudible mortar paste that presents sufficient yield stress. The material sensitivity to velocity is shown to depend on the material rheology. Experimental techniques were developed in order to follow the material evolution inside the billet. The local density evolution and the measurement of the local surface hardness of the material give sufficiently precise indications to analyse the material state of consolidation and qualify the multiphase flow of the liquid 
through the granular skeleton. The dead zone geometry and its evolution are clearly described. This study highlights the mechanism of water filtration and the consolidating zone. The material seems to consolidate layer by layer from the ram to the die as the liquid flow through the die exit. The remaining material becomes too hard to be extruded and the process is stopped. Moreover, the grains of the consolidated layers form a vault against the extruder wall opposed to the extrusion flow. We also show that part of the liquid flow toward and through the die, creating a shearing zone where the material becomes softer.

The process velocity influence is highlighted and the material heterogeneous evolution is clearly shown by the yield stress evolution in different location inside the extruder.

Due to heterogeneity and consolidation of firm mortar in extrusion flow, extruders have to be optimized to provide artificial lubrication of the extruder body and/or higher ram velocity to ensure the industrial process of such materials.

\section{References}

A.S. Burbidge, J. Bridgwater, Z. Saracevic, Liquid migration in Paste Extrusion, Transactions IchemE 73 (A) (1995) 810-816

P. Coussot, C. Ancey, Rheophysical classification of concentrated suspensions and granular pastes, Physical Review Letter E 59 (4) (1999) 4445-4456

J. Götz, H. Buggish, M. Peciar, NMR imaging of pastes in a ram extruder, Journal of Non-Newtonian Fluid Mechanics 49 (1993) 251-275

J. Götz, W. Kreibich, M. Peciar, Extrusion with a piston extruder for the determination of the local solid and fluid concentration, the local porosity and saturation and displacements profiles by means of NMR imaging, Rheologica Acta 41 (2002) 134-143

R. Hill, Mathematical theory of plasticity, Clarendon Press, Oxford, 1950

Y. Li, A.S., Burbidge, J. Bridgwater, Z. Saracevic, Liquid migration in Paste Extrusion, Transactions of the IchemE 73 (A) (1995) 810-816

B. Mu, Z. Li, S.N.C. Chui, J. Peng, Cementitious composite manufactured by extrusion technique, Cement and concrete research 29 (1999) 237-240

A. Peled, S.P. Shah, Processing effects in cementitious composites: extrusion and casting, Journal of Materials in Civil Engineering 29 (1999) 237-240

A. Perrot, C. Lanos, P. Estellé, Y. Mélinge, Ram extrusion force for a frictional plastic material: model prediction and application to cement paste, Rheologica Acta 45 (2006) 457-467

J.F. Steffe, Rheological methods in food engineering process. Second Edition, Freeman Press, East Lansing, USA, 1996

Z. Toutou, C. Lanos, Y. Mélinge, The use of squeezing test-extrusion flow similitude to identify cement based materials extrudability, Proc. XIVth Int. Congr. On Rheology, Seoul (2004)

Z. Toutou, N. Roussel, C. Lanos, The squeezing test, a tool to identify firm cement based material's rheological behaviour and evaluate their extrusion ability, Cement and Concrete Research 35 (2005) 18911899

X. Zhou, Z. Li, Characterizing rheology of fresh short fiber reinforced cementitious composite through capillary extrusion, Journal of Materials in Civil Engineering 1 (2005) 28-35

\section{Figure Caption}

Figure 1: Relationship between yield stress $\mathrm{K}$ and water content $\mathrm{w}(\%)$ for the studied mortar Figure 2: Geometry of the ram extruder and axis system

Figure 3: Protocol of the measurements of the local density

Figure 4: Protocol of the measurements of the index of consistency

Figure 5: Relationship between yield stress $\mathrm{K}$ and consistency index for the studied mortar Figure 6: (a) Local density evolution of the billet material for an extrusion performed with $d / \mathrm{D}=$ $0.35, \mathrm{~m}=600 \mathrm{~g}$ and $\mathrm{V}=0.1 \mathrm{~mm} / \mathrm{s}$ (b) Local density evolution of the billet material for an extrusion performed with $\mathrm{d} / \mathrm{D}=0.35, \mathrm{~m}=600 \mathrm{~g}$ and $\mathrm{V}=1 \mathrm{~mm} / \mathrm{s}$. 
Figure 7: Map of the surface hardness index (needle penetration force in $\mathrm{N}$ ) performed on the remaining billet of an extrusion with $\mathrm{d} / \mathrm{D}=0.35, \mathrm{~m}=600 \mathrm{~g}, \mathrm{~V}=0.1 \mathrm{~mm} / \mathrm{s}$ stopped for a ram displacement of (a) $5 \mathrm{~mm}$, (b) $15 \mathrm{~mm}$, (c) $35 \mathrm{~mm}$.

Figure 8: Map of the surface hardness performed index (needle penetration force in $\mathrm{N}$ ) on the remaining billet of an extrusion with $\mathrm{d} / \mathrm{D}=0.35, \mathrm{~m}=600 \mathrm{~g}, \mathrm{~V}=1 \mathrm{~mm} / \mathrm{s}$ stopped for a ram displacement of (a) $5 \mathrm{~mm}$, (b) $15 \mathrm{~mm}$, (c) $35 \mathrm{~mm}$, (d) $50 \mathrm{~mm}$.

Figure 9: Schematisation of the liquid relative flow in the granular skeleton

Figure 10: Opposing flow granular vault visualisation on a bi-coloured layered mortar extrusion flow. $\mathrm{d} / \mathrm{D}=0.35, \mathrm{~V}=1 \mathrm{~mm} / \mathrm{s}, \mathrm{m}=800 \mathrm{~g}$

Figure 11: Evolution of the material yield stress during extrusion (a) in the dead zone, (b) in the shearing zone, (c) at the ram. Results given for two ram velocities $(0.1$ and $1 \mathrm{~mm} / \mathrm{s}), \mathrm{d} / \mathrm{D}=0.35$ and $\mathrm{m}=600 \mathrm{~g}$.

Figure 12: Influence of the ram velocity on the flow typology of an extrusion of mortars performed with an extrusion ration of 0.35 and a billet mass of $600 \mathrm{~g}$ for two different ram velocities (a) 1 and (b) $0.1 \mathrm{~mm} / \mathrm{s}$.

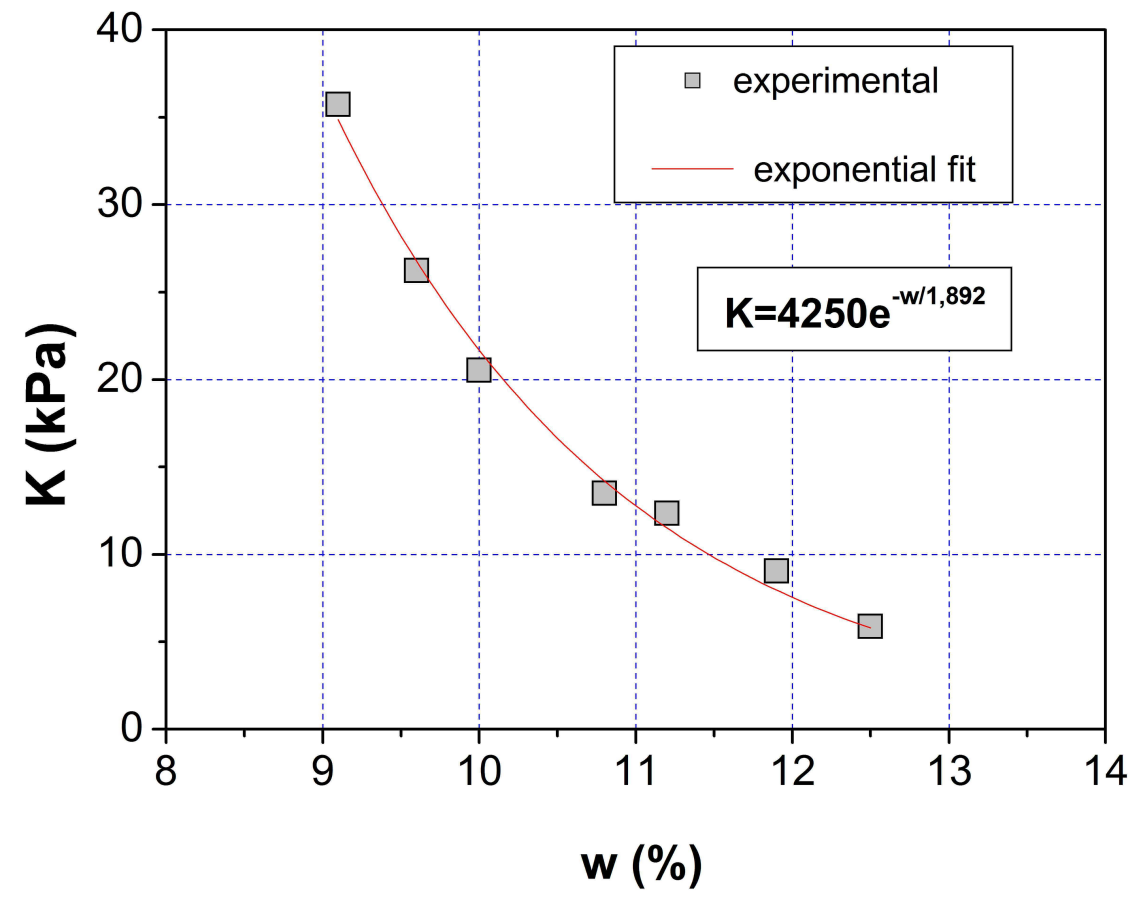

Figure 1 


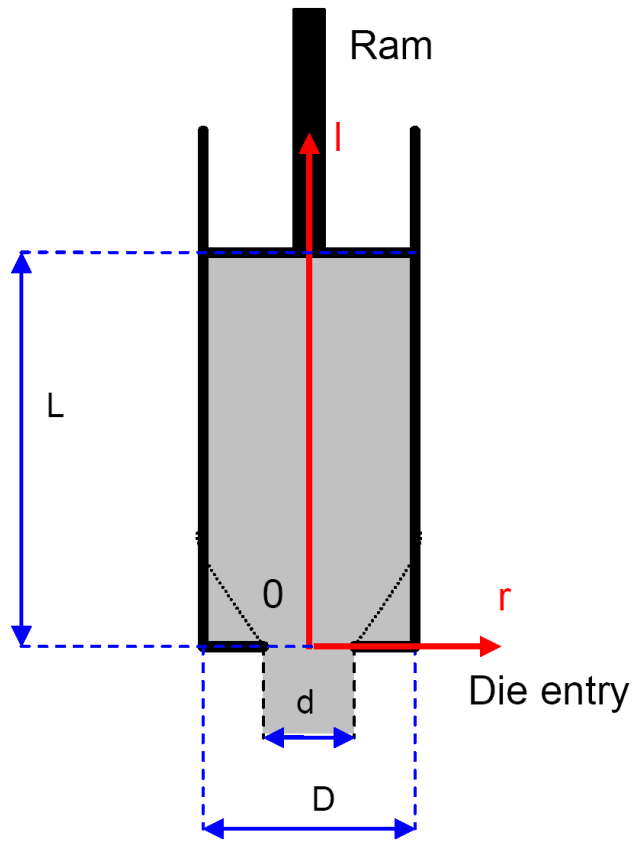

Figure 2
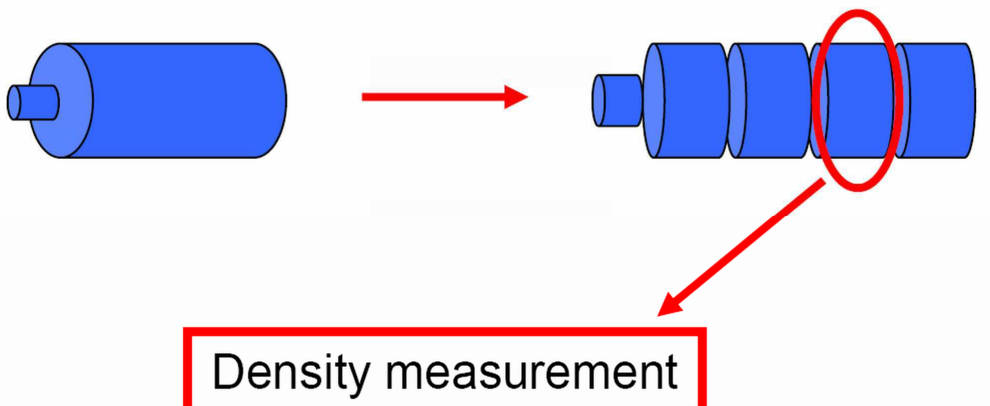

Figure 3

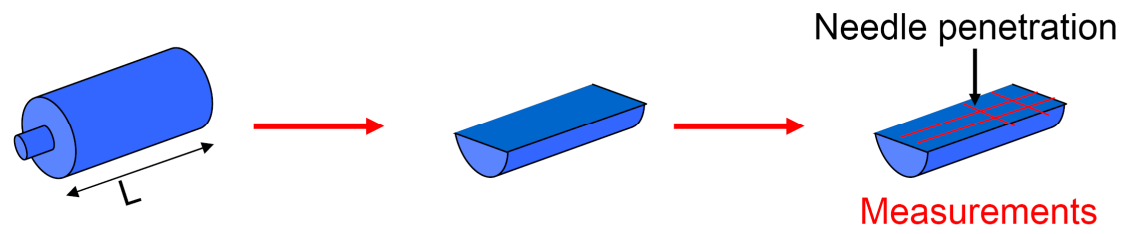

Figure 4 


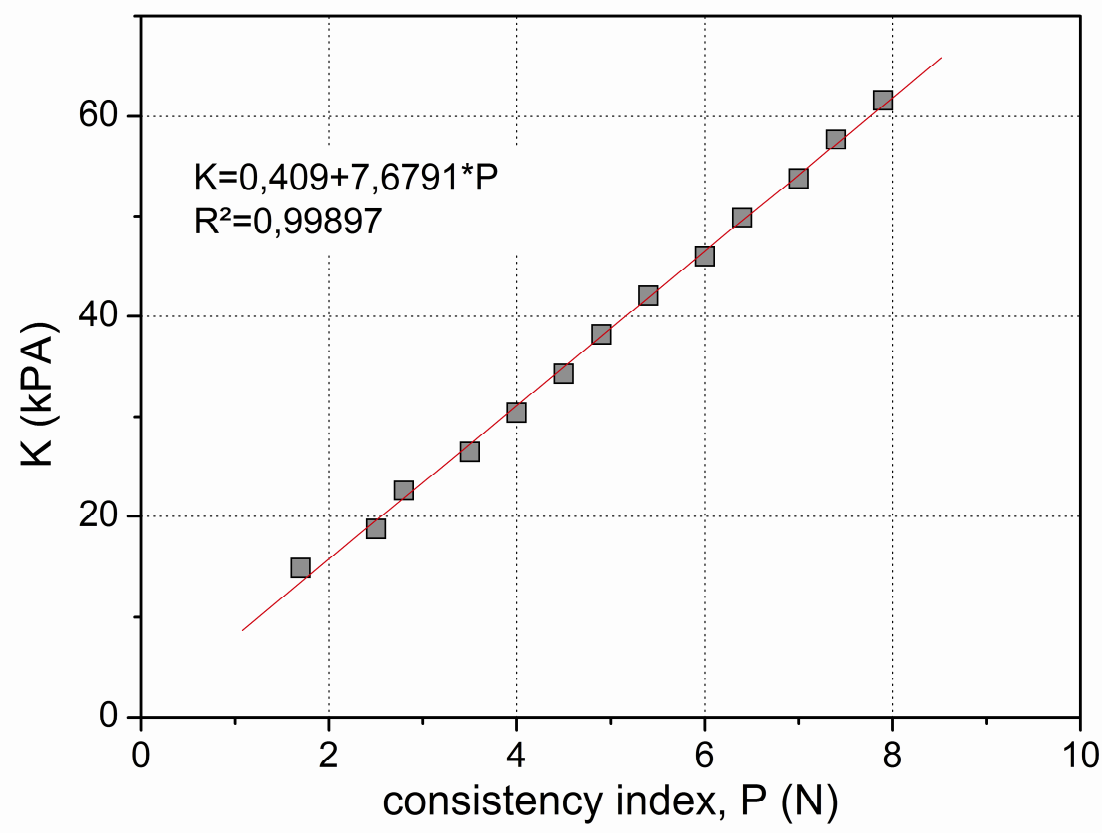

Figure 5

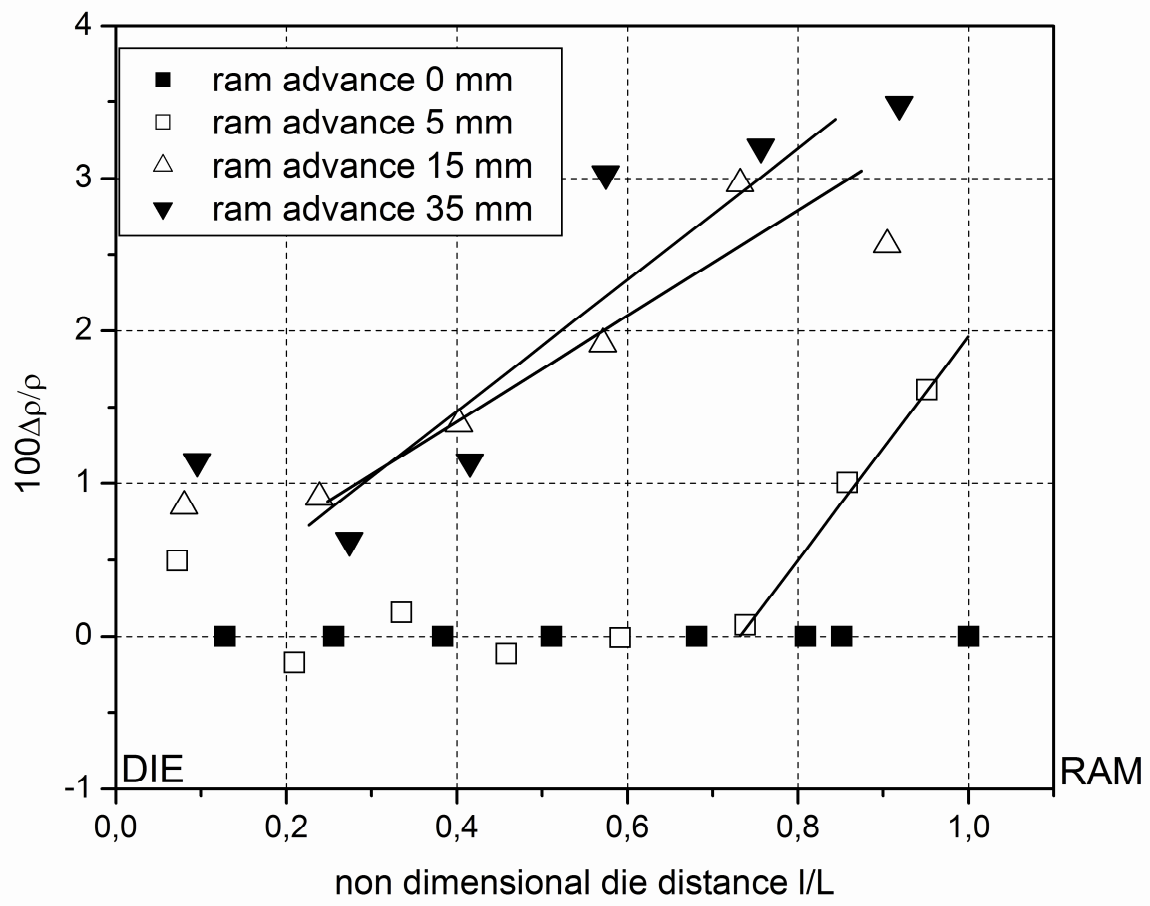

figure $6 a$ 


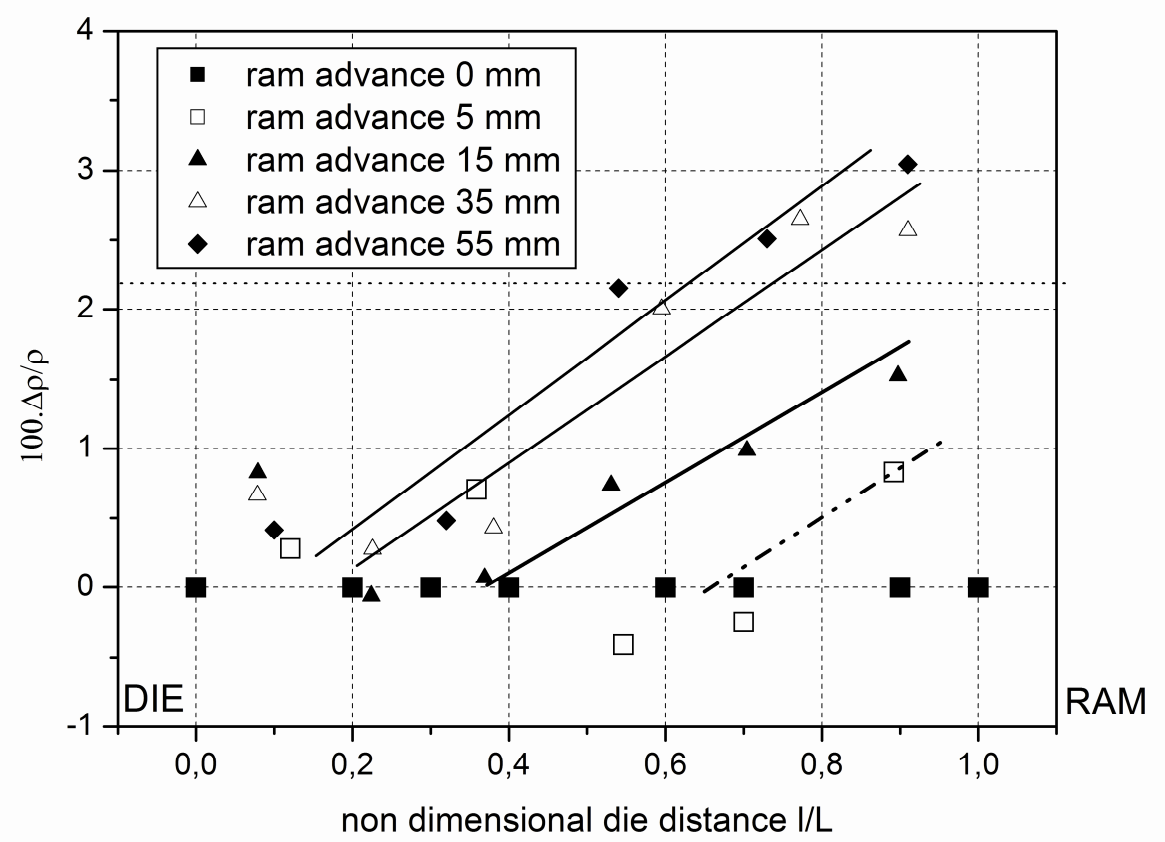

figure $6 b$

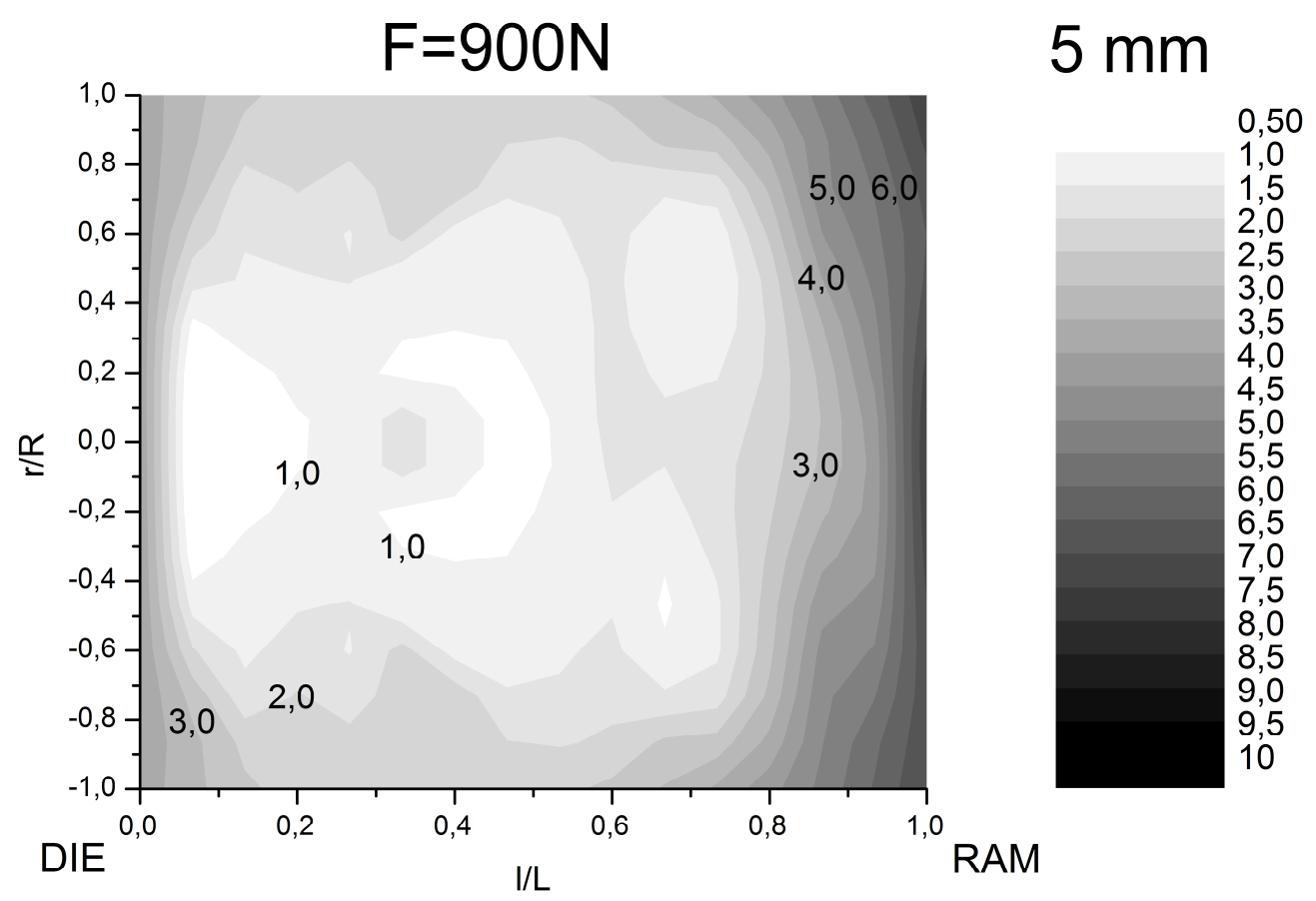

figure $7 \mathrm{a}$ 


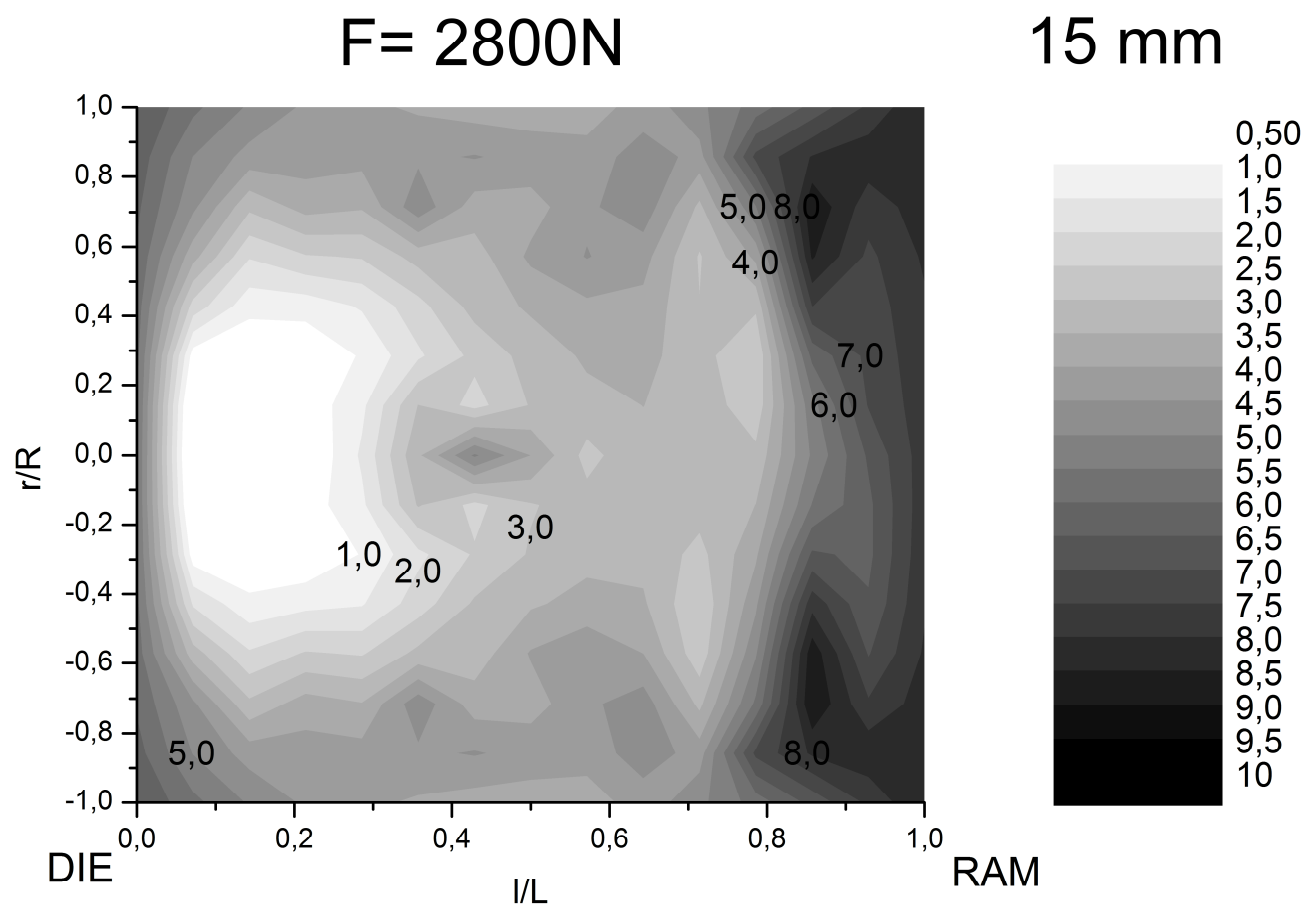

figure $7 b$

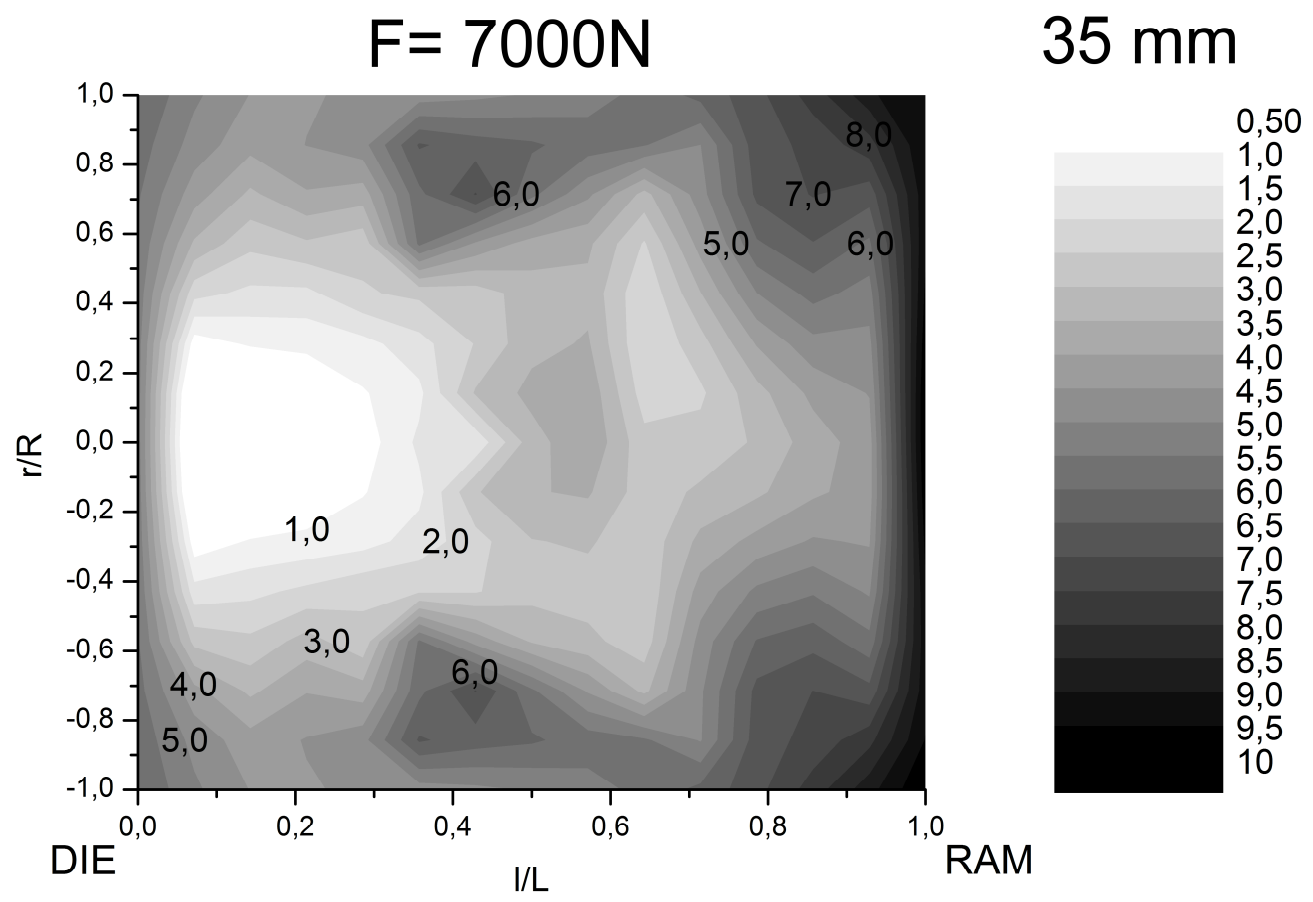

figure $7 c$ 


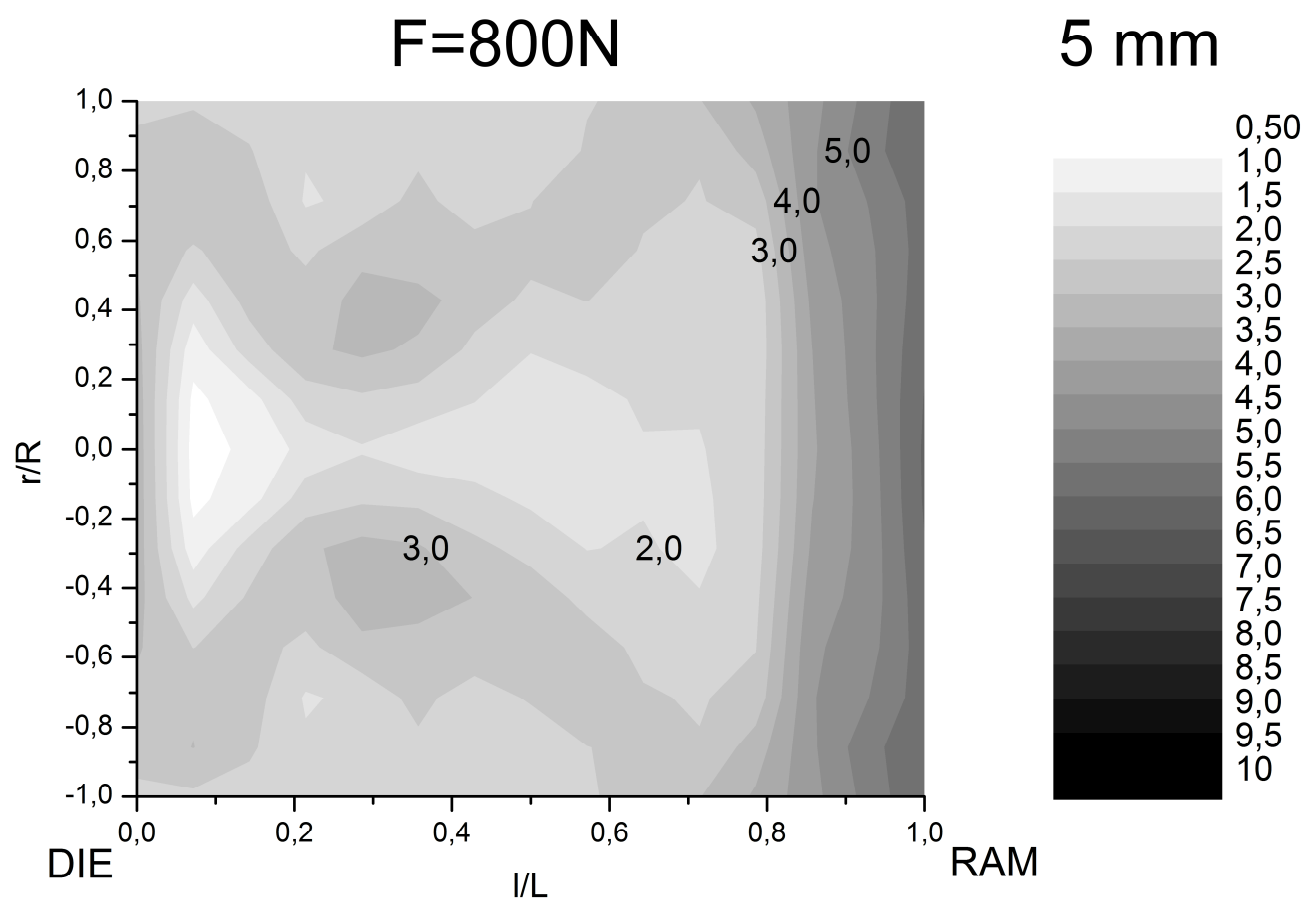

figure $8 \mathrm{a}$

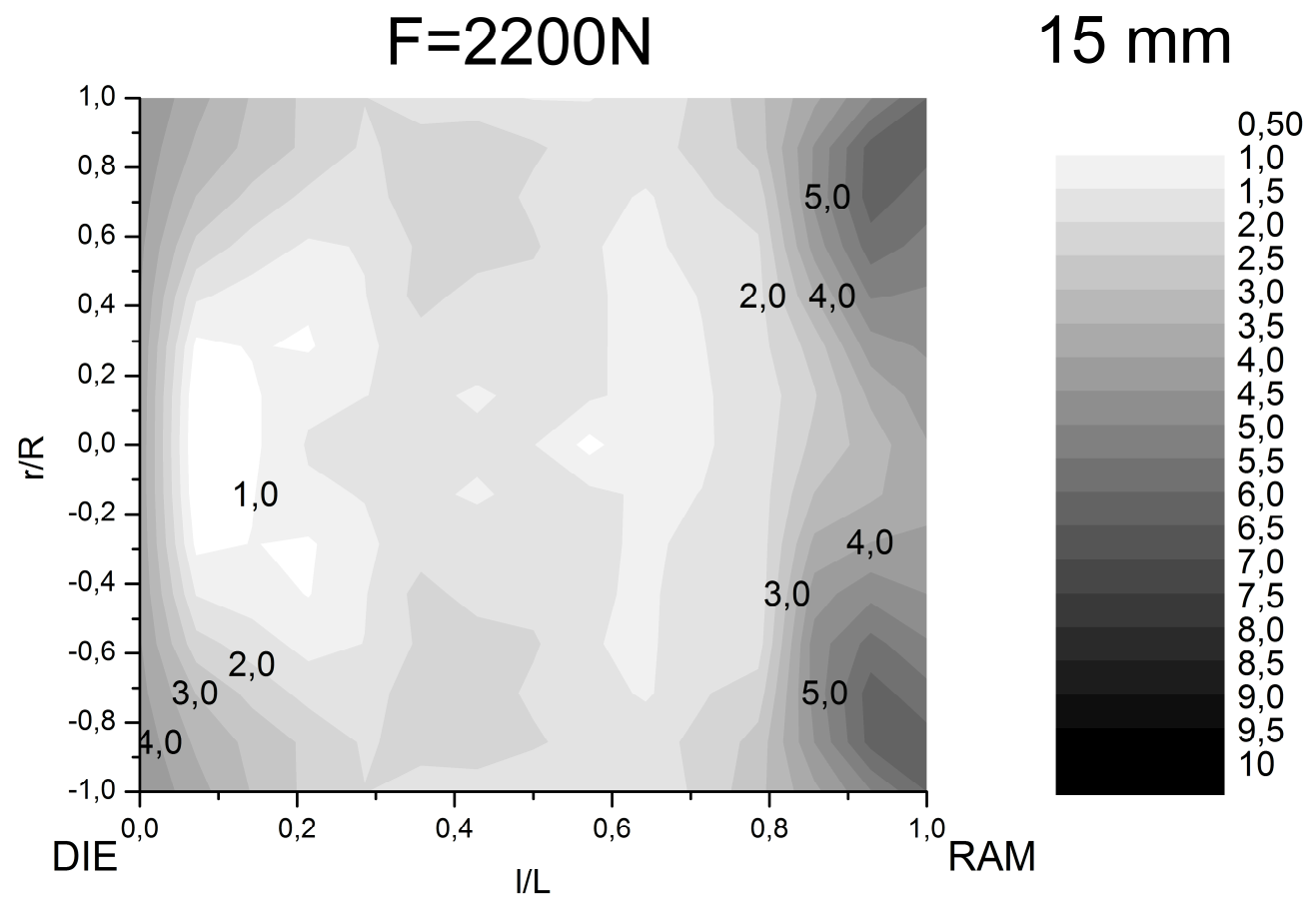

figure $8 b$ 


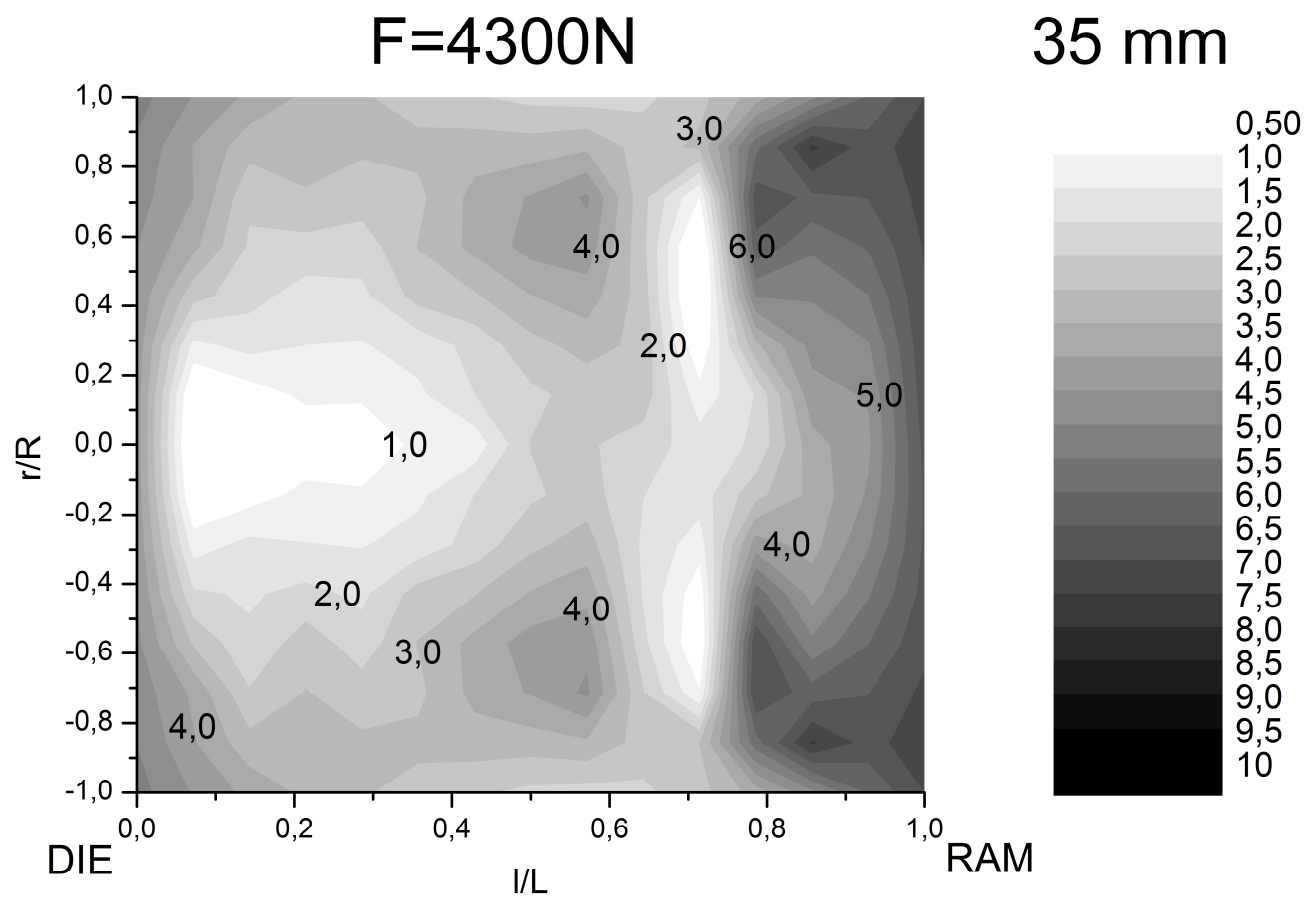

figure $8 \mathrm{c}$

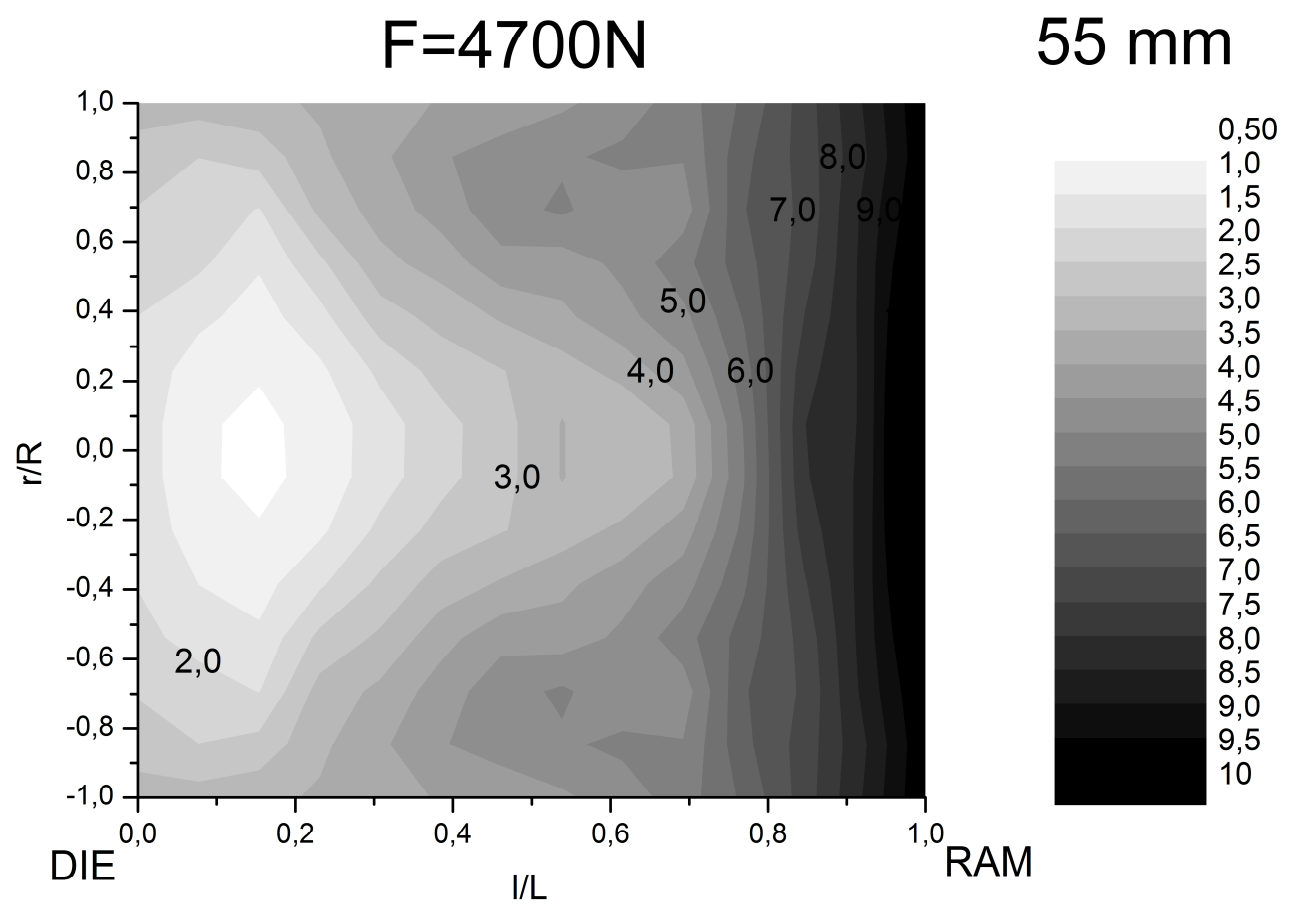

figure $8 d$

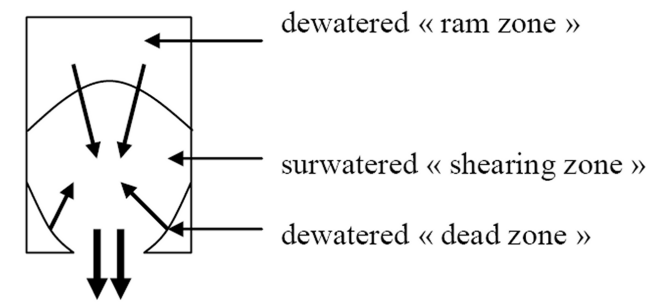

figure 9 


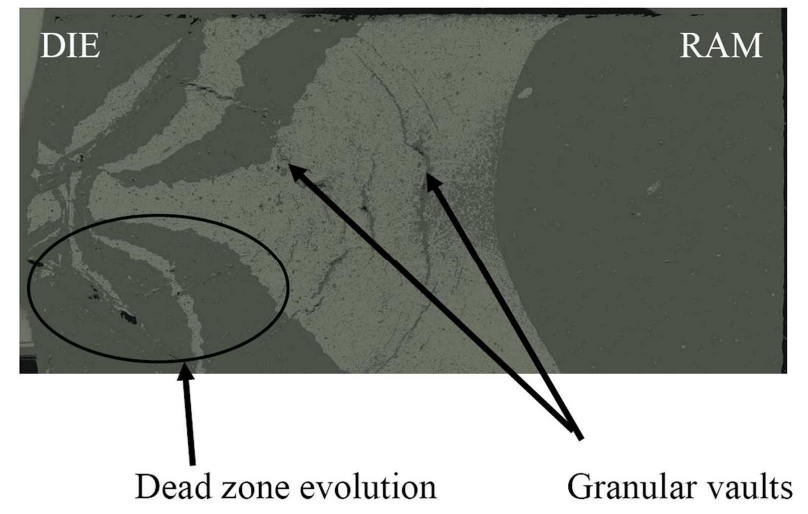

figure 10

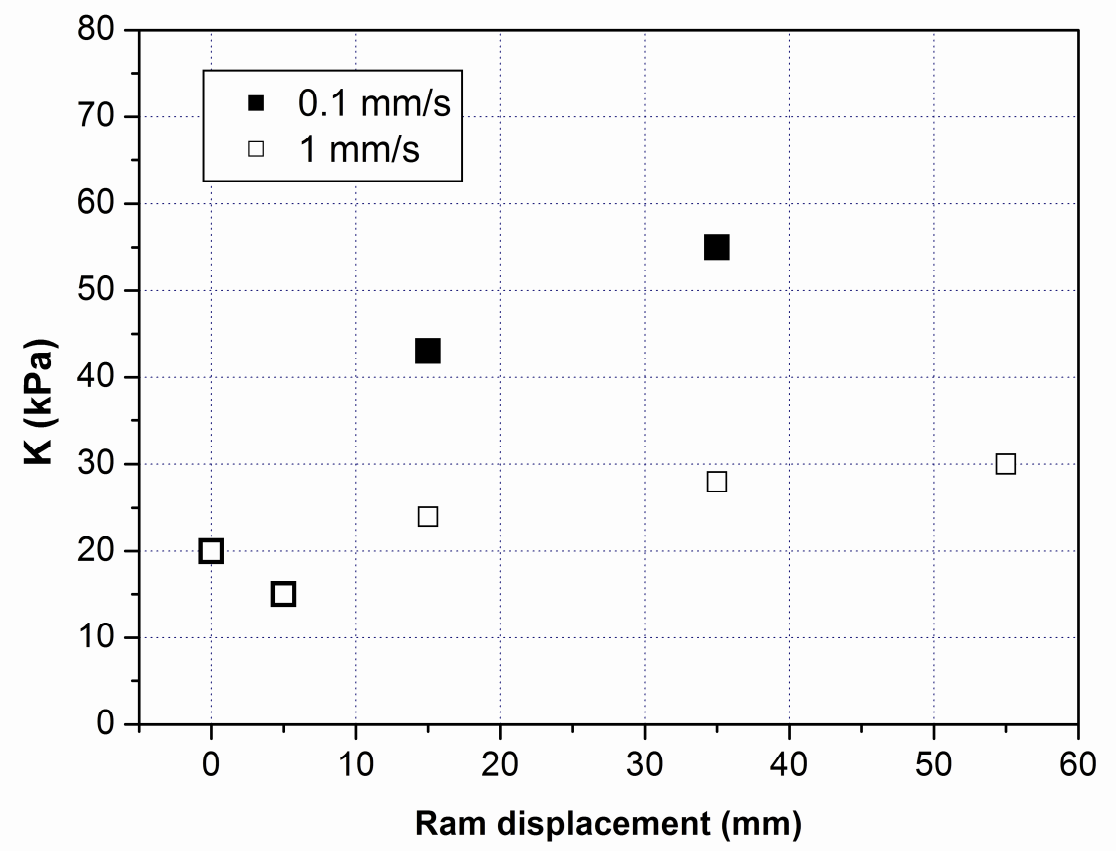

Figure 11a 


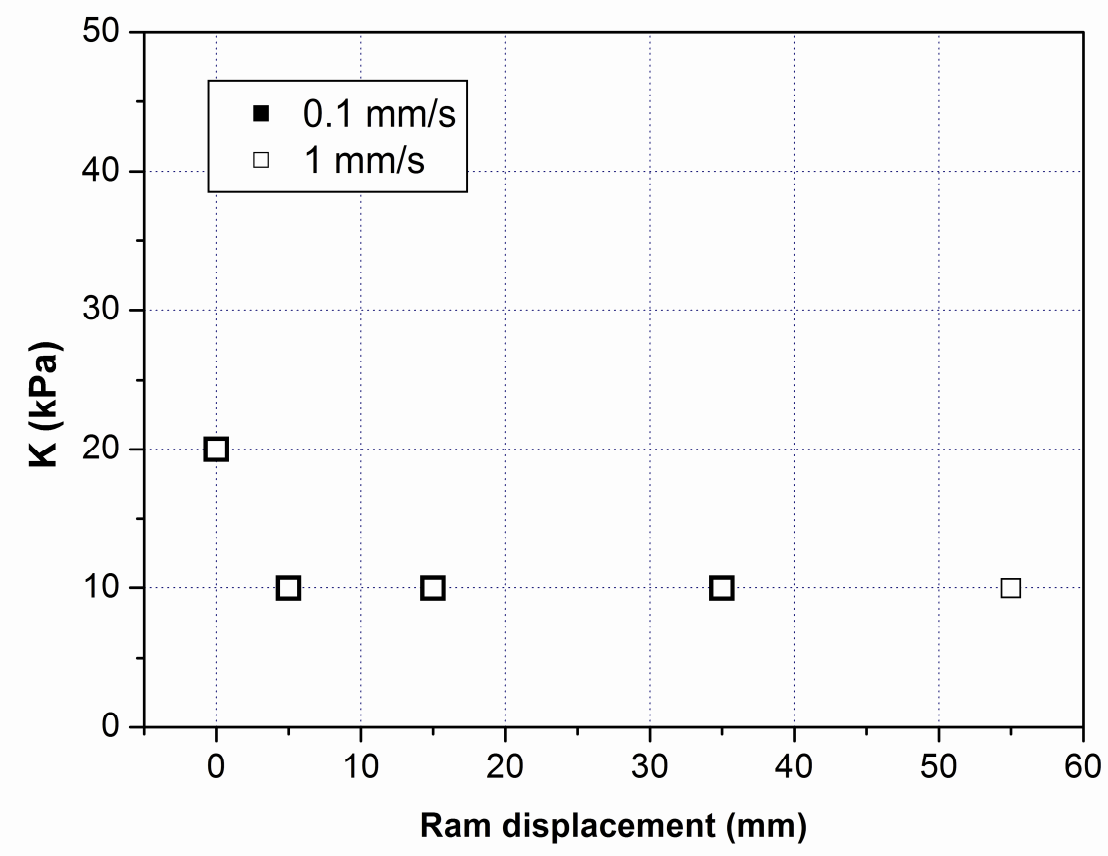

Figure $11 b$

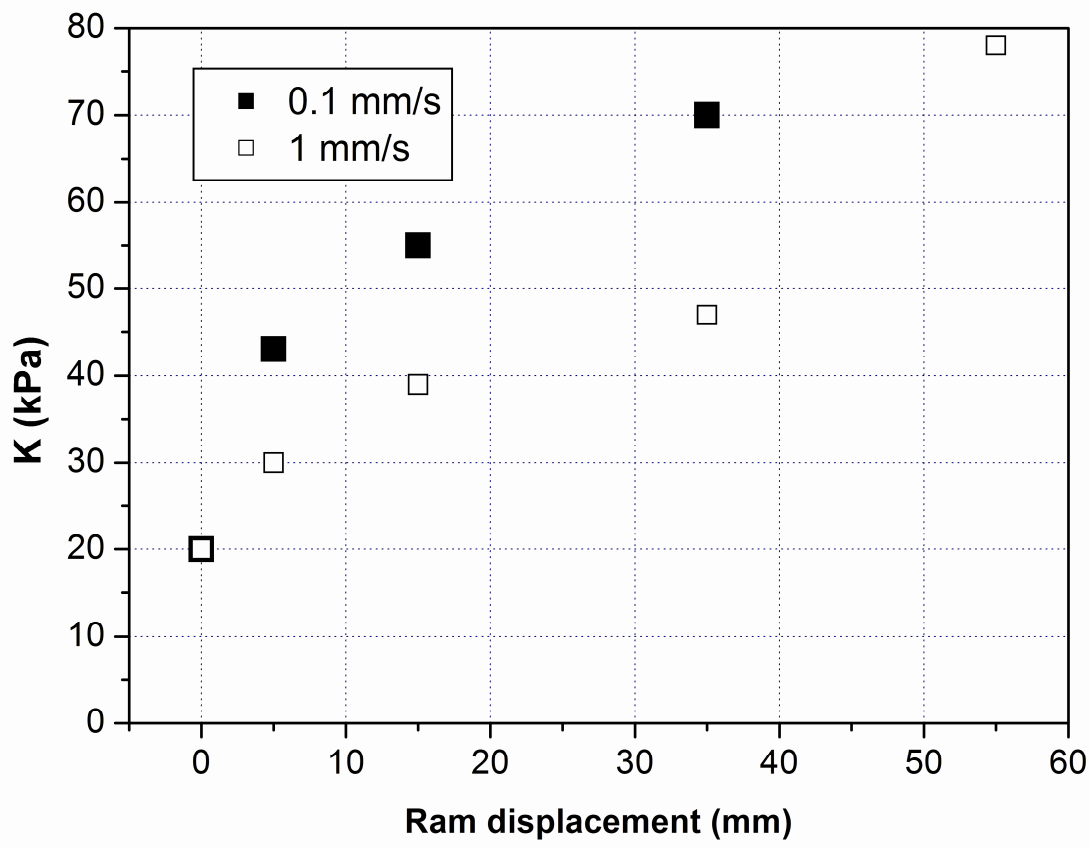

Figure 11c 


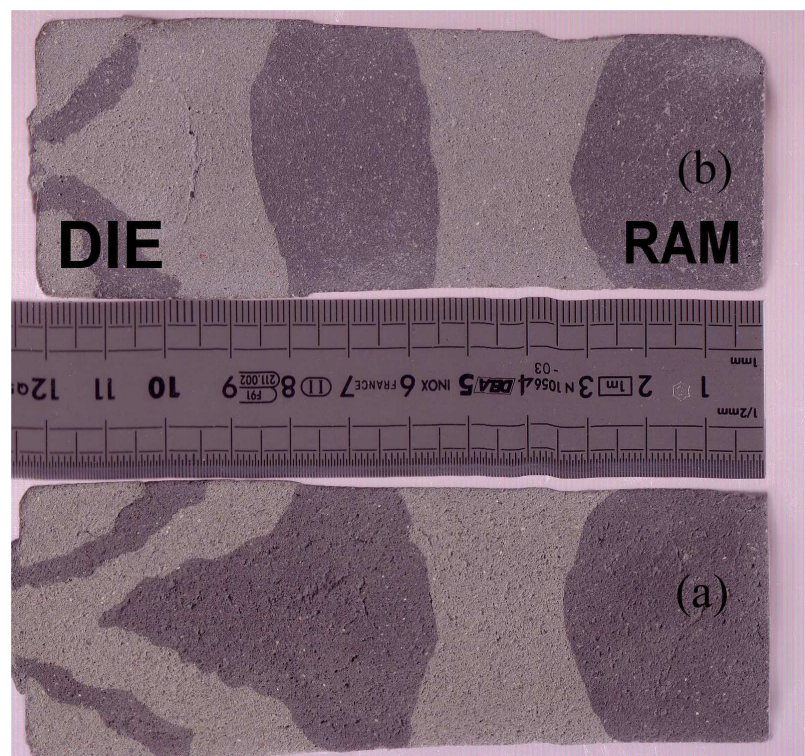

figure 12 